\title{
Analysis of formulas to calculate the AC resistance of different conductors' configurations
}

\author{
Jordi-Roger Riba \\ Escola d'Enginyeria d'Igualada, Universitat Politècnica de Catalunya, Pla de la Massa 8, 08700 Igualada, \\ Spain \\ Tel.: +34 938035300; fax: +34 938031589. E-mail address: jordi.riba@eei.upc.edu
}

\begin{abstract}
Skin and proximity effects in single- or multi-conductor systems can notoriously affect the AC resistance in conductors intended for electrical power transmission and distribution systems and for electronic devices. This increase of the AC resistance raises power loss and limits the conductors' currentcarrying capacity, being an important design parameter. There are some internationally recognized exact and approximated formulas to calculate the AC resistance of conductors, whose accuracy and applicability is evaluated in this paper. However, since these formulas can be applied under a wide range of configurations and operating conditions, it is necessary to evaluate the applicability of these models. This is done by comparing the results that they provide with experimental data and finite element method (FEM) simulation results. The results provided show that FEM results are very accurate and more general than those provided by the formulas, since FEM models can be applied to a wide range of conductors' configurations and electrical frequencies.
\end{abstract}

Keywords - Ampacity, current density, AC resistance, finite element method, power loss.

\section{INTRODUCTION}

It is well-known that although the current density distribution in homogeneous conductors supplied with direct current (DC) is uniform, when dealing with conductors under alternating current (AC) supply, their current density is often not uniform throughout the cross-section because of the skin and proximity effects $[1,2,3]$. At high frequencies the current tends to be concentrated towards the periphery of the conductor [4], thus increasing the $\mathrm{AC}$ resistance and power loss in the conductor [5]. The AC resistance is an important 
design parameter in conductors, since it allows estimating their current carrying-capacity or ampacity and operating temperature [6,7]. Therefore, the calculation of the eddy currents effects and especially the AC resistance is of paramount importance [8].

There are both exact and simplified formulas to calculate the AC resistance in conductors which are analyzed in this paper. However, analytical exact formulas are limited to a reduced number of conductors geometries and configurations [2] since they lead to challenging mathematical complications even for simple geometries [9], whereas the internationally recognized formula found in the IEC 60287-1-1 standard [10] cannot take into account some effects such as some cross-sectional geometries, multi-conductor systems with specific current distributions and/or asymmetric configurations, multiple configurations of multi-phase conductors close each other, unbalanced multi-phase conductor systems or extended frequency ranges. Contrarily, numerical methods specially conceived to deal with such type of problems tend to be more flexible, allowing to deal with multi-conductor systems and with a wider range of cross-sections and frequencies [11]. Specifically, the finite element method (FEM) is well suited for this application since it has been proven to provide accurate solutions for calculating power losses in power conductors, inductors and transformer windings [12-14]. In this paper some of the formulas found in the technical bibliography are analyzed for different conductors' configurations and their accuracy is compared with experimental data and results provided by FEM simulations. It is worth noting that regardless the importance of the practical consequences of the AC resistance in conductors, there are virtually no published technical works analyzing the accuracy of such formulas to calculate the AC resistance for different configurations, so this paper makes a contribution in this area. This paper also shows that FEM simulations offer simplicity and flexibility to analyze skin and proximity effects in single- and multi-conductor systems with different crosssectional shapes.

\section{AC RESISTANCE OF AN ISOLATED INFINITELY LONG RECTILINEAR CONDUCTOR}

The current density within an isolated conductor carrying an AC current is largest near the conductor 
surface and decreases towards its center [4] due to the skin effect. This effect is produced by the eddy currents induced by the magnetic field generated by the alternating current. The eddy currents tend to partially cancel out the AC current in the center of the conductor while reinforcing the AC current in the periphery or skin of the conductor. Therefore the current density is concentrated in the outer layers of the conductor, thus reducing the effective cross-section of the conductor and increasing its effective or AC resistance. This effect generates important practical consequences [15], such as a temperature rise, thus limiting the conductor ampacity. The skin effect becomes especially important for large conductors sections or at higher supply frequencies [16], where the skin depth $\delta$ is smaller. The skin depth in a conductor is calculated as,

$\delta=\left(\pi \cdot f \cdot \mu_{r} \cdot \mu_{0} \cdot \sigma\right)^{-1 / 2}$

$f$ being the supply frequency, $\sigma$ the electrical conductivity, $\mu_{r}$ the relative permeability of the material and $\mu_{0}$ the free space permeability. From (1) it is obvious that the skin depth diminishes for increasing values of $f, \mu_{r}$ and $\sigma$.

The $R_{a d} / R_{d c}$ resistance ratio is often calculated as an indicator of the change in the AC resistance of a conductor. To evaluate this ratio in a conductor, it is first necessary to determine the conductor DC resistance, which is determined as [10],

$R_{d c}=\frac{\rho_{20}}{S}\left[1+\alpha_{20}\left(T-T_{0}\right)\right] \quad[\Omega / \mathrm{m}]$

$T$ being the operating temperature and $\rho_{20}=1 / \sigma_{20}$ the resistivity measured at $T_{0}=20{ }^{\circ} \mathrm{C}, \alpha_{20}$ the temperature coefficient at $20^{\circ} \mathrm{C}$ and $S$ the cross-section of the conductor.

This paper assumes straight and infinitely long conductors with both uniform cross section and uniform nonmagnetic material properties.

\subsection{Round solid conductor}

Round solid conductors are widely applied in many applications including bus bars, grounding systems, power systems and in electronics and communication devices [15] among others. 
In the case of an isolated infinite long solid conductor with circular cross-section there is an internationally recognized formula that provides an exact solution of the AC resistance $[4,5]$ based on the zero-order Kelvin functions of first kind,

$$
R_{a c}=\frac{\sqrt{2}}{\pi d_{c} \sigma \delta} \cdot \frac{\operatorname{ber}(q) \cdot \operatorname{bei}^{\prime}(q)-\operatorname{bei}(q) \cdot \operatorname{ber}^{\prime}(q)}{\left[\operatorname{ber}^{\prime}(q)\right]^{2}+\left[\operatorname{bei}^{\prime}(q)\right]^{2}}[\Omega / \mathrm{m}]
$$

$d_{c}$ being the conductor diameter, $q=d_{c} /(\sqrt{2} \delta)$, ber and bei being, respectively, the real and imaginary parts of the zero-order Kelvin functions of first kind, and ber' and bei' their derivatives. The real and imaginary parts of the $n$-order Kelvin functions of first kind and their derivatives are as follows [17],

$$
\begin{aligned}
& \operatorname{ber}(n, q)=(q / 2)^{n} \sum_{k \geq 0} \frac{\cos [(3 n / 4+k / 2) \pi]}{k !(n+k) !} \cdot\left(q^{2} / 4\right)^{k} \\
& \operatorname{bei}(n, q)=(q / 2)^{n} \sum_{k \geq 0} \frac{\sin [(3 n / 4+k / 2) \pi]}{k !(n+k) !} \cdot\left(q^{2} / 4\right)^{k} \\
& \operatorname{ber}^{\prime}(n, q)=\frac{\operatorname{ber}(n+1, q)+\operatorname{bei}(n+1, q)}{\sqrt{2}}+(n / q) \cdot \operatorname{ber}(n, q) \\
& \operatorname{bei}^{\prime}(n, q)=\frac{\operatorname{bei}(n+1, q)-\operatorname{ber}(n+1, q)}{\sqrt{2}}+(n / q) \cdot \operatorname{bei}(n, q)
\end{aligned}
$$

By carefully analyzing (3), one can observe that the series expansions in (4)-(7) can produce an arithmetic overflow of the computer capacity for large $q$ values.

Since equation (3) is not straightforward to apply, in the technical literature one can find different approximate formulas derived from (3), such as the one derived by Al-Asadi et al. [4],

$$
R_{a c}=\frac{1}{\pi \sigma \delta\left(1-e^{-r / \delta}\right) \cdot\left[2 r-\delta\left(1-e^{-r / \delta}\right)\right]}[\Omega / \mathrm{m}]
$$

where $r$ is the conductor radius.

The most widely applied approximated formula to calculate the AC resistance is probably the one found in the IEC 60287-1-1 international standard [10], which calculates the AC resistance from the DC resistance as,

$$
R_{a c}=R_{d c}\left(1+y_{s}\right)[\Omega / \mathrm{m}]
$$


The skin effect factor $y_{s}$ is calculated as,

$y_{s}=\frac{x_{s}^{4}}{192+0.8 x_{s}^{4}}$

where,

$x_{s}^{4}=\left(\frac{8 \pi f K_{s}}{R_{d c} 10^{7}}\right)^{2}$

and $K_{s}=1$ in the case of a solid round conductor. However, according to [10], to obtain accurate results

(9) is only applicable when $x_{s} \leq 2.8$.

\subsection{Tubular round conductor}

When dealing with solid conductors with large cross-sections, the skin effect can be very important even at power frequency, thus significantly increasing its $\mathrm{AC}$ resistance, voltage drop and power loss. Therefore tubular conductors can partially reduce these drawbacks, while optimizing conductor weight and cost. The applications of tubular conductors are found in outdoors substation busbars, MRI devices, particle accelerators or induction furnaces among others.

There is an exact solution for the AC resistance of an insulated infinitely long round circular conductor based on the zero-order Kelvin functions of first kind (ber, bei) and second kind (ker, kei) and their derivatives (ber',bei' and ker', kei') [2],

$R_{a c}=\operatorname{real}\left[\frac{j \cdot m\left(r_{2}^{2}-r_{1}^{2}\right)}{2 r_{2}} \cdot \frac{\operatorname{ber}\left(m \cdot r_{2}\right)+j \cdot b e i\left(m r_{2}\right)-C \cdot k \operatorname{ker}\left(m \cdot r_{2}\right)-j C \cdot k e i\left(m \cdot r_{2}\right)}{b e r^{\prime}\left(m \cdot r_{2}\right)+j \cdot b e i^{\prime}\left(m r_{2}\right)-C \cdot k e r^{\prime}\left(m \cdot r_{2}\right)-j C \cdot k e i^{\prime}\left(m \cdot r_{2}\right)}\right] \cdot R_{d c}[\Omega / \mathrm{m}](12)$

$r_{l}$ and $r_{2}$ being, respectively, the inside and outside radius of the tubular conductor, $m=\sqrt{2 \pi f} \mu_{0} \mu_{r} \sigma$ and $C=\left[\operatorname{ber}^{\prime}\left(m \cdot r_{1}\right)+j \cdot b e i^{\prime}\left(m r_{1}\right)\right] /\left[\operatorname{ker}^{\prime}\left(m \cdot r_{1}\right)+j \cdot k e i^{\prime}\left(m r_{1}\right)\right]$. The real and imaginary parts of the $n$-order Kelvin functions of second kind, $\operatorname{ker}(n, x)$ and $\operatorname{kei}(n, x)$ respectively, and their derivatives have the series expansion given by, 


$$
\begin{aligned}
k e r(n, x)= & 0.5(0.5 x)^{-n} \sum_{k=0}^{n-1} \frac{(n-k-1) !}{k !} \cos (0.75 n \pi+0.5 k \pi)\left(0.25 x^{2}\right)^{k}-\ln (0.5 x) \cdot \operatorname{ber}(n, x)+0.25 \pi \text { bei }(n, x) \\
& +0.5(0.5 x)^{n} \sum_{k \geq 0} \frac{\Psi(k+1)+\Psi(n+k+1)}{k !(n+k) !} \cos (0.75 n \pi+0.5 k \pi)\left(0.25 x^{2}\right)^{k}
\end{aligned}
$$$$
k e i(n, x)=-0.5(0.5 x)^{-n} \sum_{k=0}^{n-1} \frac{(n-k-1) !}{k !} \sin (0.75 n \pi+0.5 k \pi)\left(0.25 x^{2}\right)^{k}-\ln (0.5 x) \cdot \operatorname{bei}(n, x)+0.25 \pi \text { ber }(n, x)
$$$$
+0.5(0.5 x)^{n} \sum_{k \geq 0} \frac{\Psi(k+1)+\Psi(n+k+1)}{k !(n+k) !} \sin (0.75 n \pi+0.5 k \pi)\left(0.25 x^{2}\right)^{k}
$$

$\operatorname{ker}^{\prime}(n, x)=\frac{\operatorname{ker}(n+1, q)+\operatorname{kei}(n+1, q)}{\sqrt{2}}+(n / q) \cdot \operatorname{ker}(n, q)$

$\operatorname{kei}^{\prime}(n, q)=[\operatorname{kei}(n+1, q)-\operatorname{ker}(n+1, q)] / \sqrt{2}+(n / q) \cdot \operatorname{kei}(n, q)$

$\Psi$ being the digamma function, which for positive integer numbers can be calculated as,

$\Psi(x)=-0.5772157+\sum_{k=1}^{x-1} 1 / k$

The approximated formula of the IEC 60287-1-1 standard [10] also provides a solution for tubular round conductors. In this case the factor $K_{s}$ in (11) must be calculated as,

$K_{s}=\frac{\left(r_{2}-r_{1}\right) \cdot\left(r_{2}+2 r_{1}\right)^{2}}{\left(r_{2}+r_{1}\right)^{3}}$

\section{AC RESISTANCE OF PARALLEL INFINITELLY LONG RECTILINEAR CONDUCTORS}

When two or more conductors are close each other, the AC magnetic field generated by each one affects the neighboring conductors, thus inducing eddy currents in such conductors. As a consequence, the electric current distribution within the conductors will change when compared with that of an isolated conductor. This phenomenon is known as proximity effect, and jointly with the skin effect increases both the AC resistance and power loss of the conductor since the current is confined within a region of the conductor cross-section.

Arnold [18] suggested an approximate formula to calculate the AC to DC resistance ratio of two parallel conductors of circular cross-section carrying currents of opposite polarity, 
$R_{a c}=R_{d c}\left[1+F(x)+\frac{a^{2} G(x)}{1-\alpha^{2} A(x)-\alpha^{4} B(x)-\alpha^{9} C(x)}\right][\Omega / \mathrm{m}]$

where $\alpha=d_{c} / s$ is the ratio between the conductors' diameter and the spacing between conductors' axes,

$x=2\left(2 \pi f 10^{-7} / R_{d c}\right)^{1 / 2}$ and the coefficients of (19) are as follows,

$F(x)=\left\{\begin{array}{c}\frac{5 x^{4}}{960+4 x^{4}} \text { if } x<2.2 \\ \text { Table } 1 \text { if } x \geq 2.2\end{array}\right.$

$G(x)=\left\{\begin{array}{c}\frac{11 x^{4}}{704+20 x^{4}} \text { if } x<1.7 \\ \text { Table } 1 \text { if } x \geq 1.7\end{array}\right.$

$A(x)=\left\{\begin{array}{c}\frac{1}{24}+\frac{8 x^{4}}{700+19 x^{4}} x<1.7 \\ \text { Table } 1 \text { if } x \geq 1.7\end{array}\right.$

$B(x)=\left\{\begin{array}{c}0 \text { if } x<1.4 \\ \text { Table } 1 \text { if } x \geq 1.4\end{array}\right.$

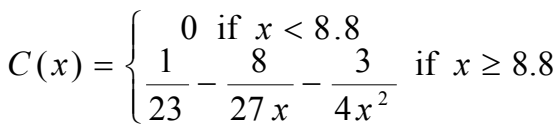

However, strictly speaking, (19) isn't an exact formula since coefficients $F(x), G(x), A(x)$ and $B(x)$ rely on the values tabulated in Table 1 .

Table 1

Values of coefficients $F(x), G(x), A(x)$ and $B(x)$

\begin{tabular}{ccccc|ccccc}
\hline $\boldsymbol{x}$ & $\boldsymbol{F}(\boldsymbol{x})$ & $\boldsymbol{G}(\boldsymbol{x})$ & $\boldsymbol{A}(\boldsymbol{x})$ & $\boldsymbol{B}(\boldsymbol{x})$ & $\boldsymbol{x}$ & $\boldsymbol{F}(\boldsymbol{x})$ & $\boldsymbol{G}(\boldsymbol{x})$ & $\boldsymbol{A}(\boldsymbol{x})$ & $\boldsymbol{B}(\boldsymbol{x})$ \\
\hline 1.30 & & & & $(23)$ & 3.55 & 0.5104 & 0.5073 & 0.4219 & -0.0036 \\
1.40 & & & & -0.0010 & 3.60 & 0.5288 & 0.5160 & 0.4275 & -0.0030 \\
1.50 & & $(21)$ & $(22)$ & -0.0018 & 3.65 & 0.5473 & 0.5247 & 0.4329 & -0.0024 \\
1.60 & & & & -0.0025 & 3.70 & 0.5659 & 0.5333 & 0.4382 & -0.0018 \\
1.70 & & 0.1055 & 0.1196 & -0.0034 & 3.75 & 0.5845 & 0.5418 & 0.4433 & -0.0012 \\
1.75 & & 0.1158 & 0.1272 & -0.0039 & 3.80 & 0.6031 & 0.5503 & 0.4482 & -0.0006 \\
1.80 & & 0.1265 & 0.1352 & -0.0043 & 3.85 & 0.6218 & 0.5588 & 0.4530 & 0.0001 \\
1.85 & $(20)$ & 0.1375 & 0.1435 & -0.0047 & 3.90 & 0.6405 & 0.5673 & 0.4577 & 0.0008 \\
1.90 & & 0.1489 & 0.1520 & -0.0052 & 3.95 & 0.6592 & 0.5758 & 0.4622 & 0.0015 \\
1.95 & & 0.1605 & 0.1608 & -0.0056 & 4.00 & 0.6779 & 0.5842 & 0.4665 & 0.0022 \\
2.00 & & 0.1724 & 0.1698 & -0.0060 & 4.05 & 0.6966 & 0.5926 & 0.4707 & 0.0029 \\
2.05 & & 0.1845 & 0.1789 & -0.0064 & 4.10 & 0.7152 & 0.6010 & 0.4748 & 0.0036 \\
2.10 & & 0.1967 & 0.1882 & -0.0068 & 4.15 & 0.7338 & 0.6094 & 0.4788 & 0.0044 \\
2.15 & & 0.2090 & 0.1976 & -0.0071 & 4.20 & 0.7523 & 0.6179 & 0.4827 & 0.0052 \\
2.20 & 0.1113 & 0.2214 & 0.2070 & -0.0074 & 4.30 & 0.7893 & 0.6348 & 0.4900 & 0.0067 \\
2.22 & 0.1150 & 0.2264 & 0.2108 & -0.0075 & 4.40 & 0.8261 & 0.6518 & 0.4969 & 0.0083 \\
2.24 & 0.1188 & 0.2313 & 0.2146 & -0.0076 & 4.50 & 0.8627 & 0.6688 & 0.5034 & 0.0098
\end{tabular}




\begin{tabular}{|c|c|c|c|c|c|c|c|c|c|}
\hline 2.26 & 0.1227 & 0.2363 & 0.2184 & -0.0078 & 4.60 & 0.8991 & 0.6858 & 0.5095 & 0.0114 \\
\hline 2.28 & 0.1267 & 0.2412 & 0.2222 & -0.0079 & 4.70 & 0.9353 & 0.7030 & 0.5152 & 0.0130 \\
\hline 2.30 & 0.1307 & 0.2462 & 0.2260 & -0.0080 & 4.80 & 0.9713 & 0.7203 & 0.5206 & 0.0146 \\
\hline 2.32 & 0.1348 & 0.2511 & 0.2298 & -0.0081 & 4.90 & 1.0071 & 0.7376 & 0.5258 & 0.0161 \\
\hline 2.34 & 0.1390 & 0.2561 & 0.2336 & -0.0082 & 5.00 & 1.0427 & 0.7550 & 0.5306 & 0.0176 \\
\hline 2.36 & 0.1433 & 0.2610 & 0.2373 & -0.0083 & 5.10 & 1.0781 & 0.7727 & 0.5351 & 0.0191 \\
\hline 2.38 & 0.1477 & 0.2659 & 0.2411 & -0.0084 & 5.20 & 1.1135 & 0.7904 & 0.5395 & 0.0205 \\
\hline 2.40 & 0.1521 & 0.2708 & 0.2449 & -0.0085 & 5.30 & 1.1487 & 0.8081 & 0.5436 & 0.0219 \\
\hline 2.42 & 0.1566 & 0.2756 & 0.2486 & -0.0086 & 5.40 & 1.1839 & 0.8258 & 0.5476 & 0.0233 \\
\hline 2.44 & 0.1612 & 0.2805 & 0.2523 & -0.0087 & 5.50 & 1.2189 & 0.8435 & 0.5514 & 0.0247 \\
\hline 2.46 & 0.1659 & 0.2853 & 0.2561 & -0.0088 & 5.60 & 1.2539 & 0.8613 & 0.5551 & 0.0260 \\
\hline 2.48 & 0.1716 & 0.2901 & 0.2598 & -0.0088 & 5.70 & 1.2889 & 0.8790 & 0.5587 & 0.0273 \\
\hline 2.50 & 0.1754 & 0.2949 & 0.2635 & -0.0089 & 5.80 & 1.3238 & 0.8967 & 0.5621 & 0.0286 \\
\hline 2.52 & 0.1803 & 0.2996 & 0.2672 & -0.0089 & 5.90 & 1.3587 & 0.9144 & 0.5654 & 0.0299 \\
\hline 2.54 & 0.1853 & 0.3043 & 0.2709 & -0.0090 & 6.00 & 1.3936 & 0.9322 & 0.5686 & 0.0311 \\
\hline 2.56 & 0.1903 & 0.3090 & 0.2745 & -0.0090 & 6.20 & 1.4634 & 0.9676 & 0.5746 & 0.0333 \\
\hline 2.58 & 0.1964 & 0.3137 & 0.2782 & -0.0090 & 6.40 & 1.5332 & 1.0031 & 0.5802 & 0.0353 \\
\hline 2.60 & 0.2006 & 0.3184 & 0.2818 & -0.0091 & 6.60 & 1.6031 & 1.0385 & 0.5855 & 0.0372 \\
\hline 2.62 & 0.2059 & 0.3230 & 0.2854 & -0.0091 & 6.80 & 1.6731 & 1.0740 & 0.5904 & 0.0390 \\
\hline 2.64 & 0.2112 & 0.3276 & 0.2889 & -0.0091 & 7.00 & 1.7432 & 1.1094 & 0.5951 & 0.0408 \\
\hline 2.66 & 0.2166 & 0.3321 & 0.2925 & -0.0091 & 7.20 & 1.8133 & 1.1448 & 995 & 0.0424 \\
\hline 2.68 & 0.2220 & 0.3367 & 0.2960 & -0.0091 & 7.40 & 1.8836 & 1.1802 & 0.6037 & 0.0439 \\
\hline 2.70 & 0.2275 & 0.3412 & 0.2995 & -0.0090 & 7.60 & 1.9538 & 1.2157 & 0.6077 & 0.0454 \\
\hline 2.75 & 0.2417 & 0.3523 & 0.3081 & -0.0090 & 7.80 & 2.0241 & 1.2511 & 0.6114 & 0.0468 \\
\hline 2.80 & 0.2562 & 0.3632 & 0.3166 & -0.0090 & 8.00 & 2.0945 & 1.2865 & 0.6149 & 0.0481 \\
\hline 2.85 & 0.2711 & 0.3739 & 0.3249 & -0.0089 & 8.20 & 2.1648 & 1.3219 & 0.6183 & 0.0493 \\
\hline 2.90 & 0.2864 & 0.3844 & 0.3330 & -0.0087 & 8.40 & 2.2352 & 1.3573 & 0.6215 & 0.0505 \\
\hline 2.95 & 0.3021 & 0.3948 & 0.3409 & -0.0085 & 8.60 & 2.3056 & 1.3928 & 0.6246 & 0.0516 \\
\hline 3.00 & 0.3181 & 0.4050 & 0.3487 & -0.0083 & 8.80 & 2.3760 & 1.4282 & 0.6275 & 0.0526 \\
\hline 3.05 & 0.3344 & 0.4150 & 0.3563 & -0.0080 & 9.00 & 2.4464 & 1.4636 & 0.6302 & 0.0536 \\
\hline 3.10 & 0.3510 & 0.4248 & 0.3636 & -0.0077 & 9.20 & 2.5168 & 1.4990 & 0.6329 & 0.0545 \\
\hline 3.15 & 0.3679 & 0.4345 & 0.3708 & -0.0074 & 9.40 & 2.5872 & 1.5344 & 0.6354 & 0.0555 \\
\hline 3.20 & 0.3850 & 4440 & 0.3779 & -0.0070 & 9.60 & 2.6577 & 1.5698 & 0.6378 & 0.0564 \\
\hline 3.25 & 0.4024 & 0.4533 & 0.3847 & -0.0066 & 9.80 & 2.7281 & 1.6052 & 0.6402 & 0.0572 \\
\hline 3.30 & 0.4200 & 0.4626 & 0.3914 & -0.0062 & 10.00 & 2.7986 & 1.6406 & 0.6424 & 0.0580 \\
\hline 3.35 & 0.4378 & 0.4717 & 0.3978 & -0.0057 & 10.50 & 2.9748 & 1.7291 & 0.6476 & 0.0599 \\
\hline 3.40 & 0.4557 & 0.4808 & 0.4041 & -0.0052 & 11.00 & 3.1510 & 1.8176 & 0.6523 & 0.0616 \\
\hline 3.45 & 0.4738 & 0.4897 & 0.4102 & -0.0047 & 11.50 & 3.3273 & 1.9061 & 0.6566 & 0.0631 \\
\hline 3.50 & 0.4920 & 0.4986 & 0.4161 & -0.0042 & 12.00 & 3.5036 & 1.9945 & 0.6606 & 0.0644 \\
\hline
\end{tabular}

Due to the inherent difficulty in applying (19), different approximations are used, among them

highlighting the one suggested in the IEC 60287-1-1 standard [10], which is an extension of (9),

$$
R_{a c}=R_{d c}\left(1+y_{s}+y_{p}\right)[\Omega / \mathrm{m}]
$$

where the proximity effect factor $y_{p}$ for two-core cables or for two single-core cables is calculated as,

$$
x_{p}^{4}=\left(\frac{8 \pi f K_{p}}{R_{d c} 10^{7}}\right)^{2}
$$


$y_{p}=\frac{2.9 x_{p}^{4}}{192+0.8 x_{p}^{4}}\left(d_{c} / s\right)^{2}$

$d_{c}$ being the diameter of the conductor, $s$ the distance between conductors axes and $K_{p}=1$ for copper round solid or stranded conductors and $K_{p}=0.8$ for tubular round conductors. According to the IEC standard, there are no accepted experimental results for aluminum conductors, so it proposes to use similar $K_{p}$ values than those suggested for copper conductors.

In the case of three parallel conductors carrying three-phase currents, the IEC 60287-1-1 standard suggests calculating the proximity effect factor $y_{p}$ as,

$y_{p}=\frac{x_{p}^{4}}{192+0.8 x_{p}^{4}}\left(d_{c} / s\right)^{2}\left[0.312\left(d_{c} / s\right)^{2}+\frac{1.18}{x_{p}^{4} /\left(192+0.8 x_{p}^{4}\right)+0.27}\right]$

The IEC 60287-1-1 standard recommends using $s$ as the distance between conductors' axes when the three conductors are equally spaced and $s=\left(s_{1} \cdot s_{2}\right)^{1 / 2}$ when the spacing between adjacent phases is not equal.

\section{THE FEM MODEL}

In this paper two-dimensional FEM models are applied since they are available, flexible, fast and easy to apply. FEM models can also deal with more complex geometries that those analyzed in this paper and provide very accurate solutions, even when analyzing problems with uneven current distributions due to eddy currents effects $[19,20]$. In addition, FEM models of conductors can deal with almost any crosssection shape. This paper deals with the free license two-dimensional FEMM package [21], which has been used since it is suitable for this application.

When dealing with time-harmonic problems, eddy currents may be induced in conductive regions. The constitutive relationship between the electric field intensity $E$ and the current density $J$ is,

$$
J=\sigma \cdot E
$$

In addition the magnetic flux density $B$ and the magnetic vector potential $A$ are related by,

$$
B=\nabla \wedge A
$$


The magnetic vector potential is selected with zero divergence, that is, $\nabla A=0$.

The induced electric field is governed by the equation,

$$
\nabla \wedge E=-\frac{\partial B}{\partial t}
$$

By merging (30) and (31) it results,

$$
\nabla \wedge E=-\nabla \wedge \frac{\partial A}{\partial t}
$$

In the case of 2-D problems, (32) can be integrated, resulting in,

$$
E=-\frac{\partial A}{\partial t}-\nabla V
$$

So, (29) leads to,

$$
J=-\sigma \frac{\partial A}{\partial t}-\sigma \nabla V
$$

Under the quasi-static approximation, that is, when the problem dimensions are small compared with the associated electromagnetic wavelength, the displacement current can be neglected [22], thus resulting in,

$$
\nabla \wedge H=J
$$

By replacing (30) into (35) it results,

$$
\nabla \wedge \frac{\nabla \wedge A}{\mu}=J
$$

Since $\nabla \wedge \nabla \wedge A=\nabla \nabla A-\nabla^{2} A$ and $\nabla A=0$, (36) can be rewritten as,

$$
-\frac{\nabla^{2} A}{\mu}=J
$$

By replacing (34) into (37) and considering a source current density term $J_{s}$, it results,

$$
-\frac{\nabla^{2} A}{\mu}=-\sigma \frac{\partial A}{\partial t}-\sigma \nabla V+J_{s}
$$

It is worth noting that FEMM solves (38) when the field oscillates at a given predetermined frequency. 
FEMM uses the vector potential formulation since once $A$ has been calculated, by differentiating the vector potential both fields $B$ and $H$ can be calculated.

All conductors' configurations studied in Section 5 have been analyzed by means of a two-dimensional FEM model that applies a circular air boundary far enough from the conductors, with a Dirichlet boundary condition that imposes the vector potential $A$ to be zero at the outer circular perimeter. Note that the solutions provided by the FEM simulations done in this paper are very fast since they have a low computational burden, so this method is very appealing to solve the problems under study.

FEM models deal with discretized complex geometric domains which must be divided into small elements by applying suitable mesh generation methods. For this purpose a variable number of triangular elements is considered in each model, which depends on both the dimensions of conductor and the frequency analyzed. Fig. 1 shows the mesh and the boundary conditions when analyzing two parallel rectilinear solid round conductors.

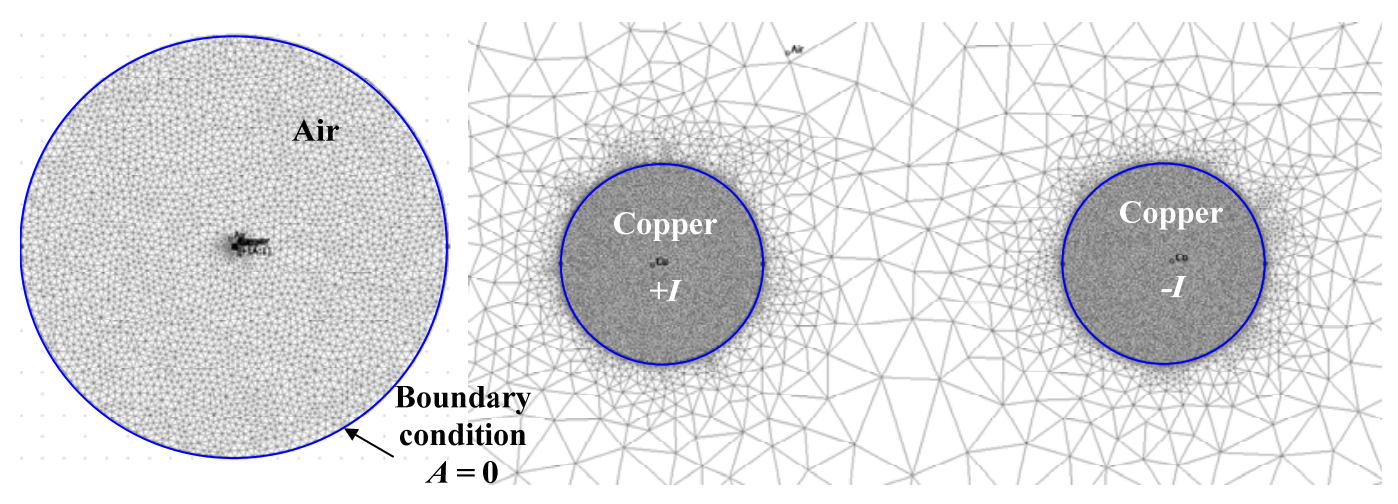

Fig. 1. Mesh example of two parallel rectilinear solid round conductors. The Dirichlet condition $A=0$ is imposed at the outer boundary.

\section{RESULTS}

This section compares the results of different conductors' configurations provided by the formulas described in Section 4 with those obtained by means of FEM simulations and experimental data found in internationally recognized publications [23-25]. 


\subsection{AC resistance of an isolated solid round conductor}

The calculation of the $R_{a d} / R_{d c}$ resistance ratio of insulated solid round conductors has been of great interest during the last century. There are some internationally recognized experimental works [23,24] whose values are analyzed in this section. These experimental results are compared with the results provided by FEM simulations, the exact equation (3), and the approximations from Al-Asadi et al. and the IEC 60287-1-1 standard, given in (8) and (9), respectively.

First, the experimental data published in [23] for a $1212 \mathrm{~mm}^{2}$ copper round conductor in the $0-100 \mathrm{~Hz}$ frequency range are compared with those provided by the exact and approximated formulas (3), (8) and (9) and FEM simulations. All these data are summarized in Table 2. Note that average relative errors shown in the following tables are calculated with respect to the measured values.

\section{Table 2}

$R_{a c} / R_{d c}$ results from exact and approximated formulas, fem simulations and experiments of a Cu conductor of $1212 \mathrm{~mm}^{2}$ and $39.28 \mathrm{~mm}$ equivalent diameter

\begin{tabular}{cccccc}
\hline $\begin{array}{c}\text { Frequency } \\
(\mathbf{H z})\end{array}$ & $\begin{array}{c}\text { Exact } \\
\mathbf{( 3 )}\end{array}$ & $\begin{array}{c}\text { Al-Asadi } \text { et al. } \\
\mathbf{( 8 )}\end{array}$ & $\begin{array}{c}\text { IEC 60287-1-1 } \\
\mathbf{( 9 )}\end{array}$ & FEM & $\begin{array}{c}\text { Measured } \\
{[23]}\end{array}$ \\
\hline 0 & 1.000 & 1.000 & 1.000 & 1.000 & 1.000 \\
25 & 1.094 & 1.298 & 1.094 & 1.094 & 1.098 \\
50 & 1.309 & 1.513 & 1.307 & 1.309 & 1.318 \\
75 & 1.544 & 1.698 & 1.528 & 1.544 & 1.550 \\
100 & 1.754 & 1.864 & 1.707 & 1.754 & 1.755 \\
\hline Average error & $\mathbf{0 . 3 0 \%}$ & $\mathbf{9 . 7 5 \%}$ & $\mathbf{1 . 0 7 \%}$ & $\mathbf{0 . 3 0 \%}$ & - \\
\hline
\end{tabular}

Results presented in Table 2 show that both the exact formula and FEM simulations produce virtually the same results, whereas the error of the approximated method proposed by the IEC 60287-1-1 raises when the frequency increases. For example, at $50 \mathrm{~Hz}$ the relative error with respect the exact formula is about $0.2 \%$ whereas at $100 \mathrm{~Hz}$ is about $2.7 \%$. Contrarily, the accuracy of (8) increases with rising values of the frequency.

Table 3 compares experimental results of the $R_{a c} / R_{d c}$ resistance ratio presented in [24] for an AWG-12 copper round conductor in the range $0-100 \mathrm{kHz}$ with those provided by formulas (3), (8), (9) and FEM simulations. The important effect that the frequency plays in the skin effect is clearly shown in the results 
summarized in Table 3. They also show that when dealing with the AWG-12 copper conductor, the formula suggested by the IEC 60287-1-1 standard becomes less accurate for frequencies above $16 \mathrm{kHz}$, since $x_{s}>$ 2.8 .

Table 3

$R_{a c} / R_{d c}$ values for an isolated solid round $\mathrm{Cu}$ conductor AWG-12 with diameter $2.052 \mathrm{~mm}$

\begin{tabular}{cccccc}
\hline $\begin{array}{c}\text { Frequency } \\
(\mathbf{H z})\end{array}$ & $\begin{array}{c}\text { Exact } \\
\mathbf{( 3 )}\end{array}$ & $\begin{array}{c}\text { Al-Asadi et al. } \\
\mathbf{( 8 )}\end{array}$ & $\begin{array}{c}\text { IEC 60287-1-1 } \\
\mathbf{( 9 )}\end{array}$ & FEM & $\begin{array}{c}\text { Measured } \\
{[\mathbf{2 4}]}\end{array}$ \\
\hline 0 & 1.000 & 1.000 & 1.000 & 1.000 & 1.000 \\
13356 & 1.185 & 1.402 & 1.184 & 1.185 & 1.185 \\
26095 & 1.509 & 1.671 & $\mathbf{1 . 4 9 7}$ & 1.509 & 1.514 \\
41726 & 1.858 & 1.950 & $\mathbf{1 . 7 8 5}$ & 1.858 & 1.860 \\
53480 & 2.071 & 2.135 & $\mathbf{1 . 9 1 8}$ & 2.071 & 2.063 \\
62977 & 2.223 & 2.274 & $\mathbf{1 . 9 9 2}$ & 2.223 & 2.214 \\
73759 & 2.381 & 2.423 & $\mathbf{2 . 0 5 1}$ & 2.381 & 2.367 \\
86149 & 2.549 & 2.583 & $\mathbf{2 . 0 9 7}$ & 2.549 & 2.537 \\
99179 & 2.714 & 2.741 & $\mathbf{2 . 1 3 1}$ & 2.714 & 2.697 \\
200000 & 3.735 & 3.744 & $\mathbf{2 . 2 1 9}$ & 3.736 & - \\
500000 & 5.748 & 5.751 & $\mathbf{2 . 2 4 5}$ & 5.749 & - \\
1000000 & 8.019 & 8.021 & $\mathbf{2 . 2 4 9}$ & 8.023 & - \\
\hline Average error & $0.33 \%$ & $5.06 \%$ & $8.22 \%$ & $0.33 \%$ & - \\
\hline
\end{tabular}

Bold numbers indicate that $x_{s}>2.8$, so (9) is inaccurate

Note that the $R_{a c} / R_{d c}$ values for the last three frequencies shown in Table 3 are not given in [24], so the error calculation does not take into account the last three frequencies.

\subsection{AC resistance of an isolated tubular round conductor}

As explained, round tubular conductors are applied in a large number of applications involving a wide interval of frequencies. In this section experimental results of a tubular copper conductor with $r_{l}=0.945$ $\mathrm{mm}$ and $r_{2}=1.590 \mathrm{~mm}$ presented in [24] are compared with those provided by the exact formula (12) and the approximated formula (9) suggested by the IEC 60287-1-1 standard and FEM simulations. These results are summarized in Table 4. 


\section{Table 4}

$R_{a c} / R_{d c}$ values for a round tubular $\mathrm{Cu}$ conductor with $r_{1}=0.945 \mathrm{~mm}$ and $r_{2}=1.590 \mathrm{~mm}$

\begin{tabular}{ccccc}
\hline $\begin{array}{c}\text { Frequency } \\
\mathbf{( H z )}\end{array}$ & $\begin{array}{c}\text { IEC 60287-1-1 } \\
\mathbf{( 9 )}\end{array}$ & $\begin{array}{c}\text { Exact } \\
\mathbf{( 1 2 )}\end{array}$ & FEM & $\begin{array}{c}\text { Measured } \\
{[\mathbf{2 4}]}\end{array}$ \\
\hline 0 & 1.000 & 1.000 & 1.000 & 1.000 \\
10864 & 1.061 & 1.056 & 1.056 & 1.054 \\
21065 & 1.201 & 1.195 & 1.195 & 1.198 \\
38420 & $\mathbf{1 . 4 8 7}$ & 1.524 & 1.524 & 1.539 \\
55340 & $\mathbf{1 . 7 1 2}$ & 1.850 & 1.850 & 1.868 \\
68360 & $\mathbf{1 . 8 3 6}$ & 2.071 & 2.071 & 2.092 \\
86375 & $\mathbf{1 . 9 5 4}$ & 2.333 & 2.333 & 2.348 \\
99405 & $\mathbf{2 . 0 1 3}$ & 2.498 & 2.498 & 2.476 \\
200000 & $\mathbf{2 . 1 8 2}$ & 3.463 & 3.464 & - \\
500000 & $\mathbf{2 . 2 3 9}$ & 5.375 & 5.377 & - \\
1000000 & $\mathbf{2 . 2 4 7}$ & Overflow & 7.538 & - \\
\hline Average error & $7.55 \%$ & $0.61 \%$ & $0.61 \%$ & - \\
\hline
\end{tabular}

Bold numbers indicate that $x_{s}>2.8$, so (9) is inaccurate

Similarly as in Table 3, the $R_{a d} / R_{d c}$ values for the last three frequencies shown in Table 4 are not given in [24], so the error calculation does not take into account the last three frequencies.

Once again, both FEM simulations and the exact formula produce almost the same results to the third decimal place and the error of the simple IEC 60287-1-1 formula grows with increasing frequency values. For example, at $10 \mathrm{kHz}$ the relative error with respect the exact formula is about $0.5 \%$ whereas at $500 \mathrm{kHz}$ it is about $54.7 \%$. In addition, for frequencies above $500 \mathrm{kHz}$, the exact formula (12) produces an arithmetic overflow of the computer capacity, so it cannot predict the AC to DC resistance ratio. This effect is well described in the technical literature [26]. When increasing the diameter of the conductor, this overflow problem will arise at lower frequencies. Therefore, it is concluded that the FEM model can accurately predict the $R_{a c} / R_{d c}$ ratio under a wide variety of conditions.

\subsection{AC resistance ratio of two parallel round solid conductors}

In the case of two neighboring rectilinear parallel conductors, the AC magnetic field generated by each one induces eddy currents in the other, thus the electric current distribution within the conductors is altered. 
This is the proximity effect, which jointly with the skin effect can severely increase the AC resistance in both conductors. It is worth noting that formulas (19) and (25) can only deal with conductors carrying AC currents with opposite polarity, whereas FEM simulations can deal with both, currents of the same and opposite polarity. This section compares the results for two parallel conductors carrying opposite AC currents provided by the approximated formulas (19) and (25) with those outputted by the FEM model and experimental measurements from [18].

Table 5 summarizes the results of such formulas for two AWG-0000 parallel solid round conductors with diameter $11.68 \mathrm{~mm}$ diameter carrying currents of opposite polarity.

\section{Table 5}

$R_{a c} / R_{d c}$ values for two parallel solid round $\mathrm{Cu}$ conductors with radius $16 \mathrm{~mm}$ spaced $32.56 \mathrm{~mm}$ and $79.45 \mathrm{~mm}$ between axes carrying AC currents of opposite polarity

\begin{tabular}{|c|c|c|c|c|}
\hline $\begin{array}{c}\text { Frequency } \\
\text { (Hz) }\end{array}$ & $\begin{array}{c}\text { IEC 60287-1-1 } \\
(25)\end{array}$ & $\begin{array}{c}\text { Arnold } \\
\text { (19) }\end{array}$ & FEM & $\begin{array}{c}\text { Measured } \\
{[18]} \\
\end{array}$ \\
\hline \multicolumn{5}{|c|}{ Spacing between axes: $32.56 \mathrm{~mm}$} \\
\hline 0 & 1.000 & 1.000 & 1.000 & 1.000 \\
\hline 24.87 & 1.163 & 1.160 & 1.159 & 1.160 \\
\hline 49.73 & 1.590 & 1.500 & 1.497 & 1.501 \\
\hline 74.56 & 2.148 & 1.870 & 1.858 & 1.870 \\
\hline 99.33 & 2.717 & 2.222 & 2.208 & 2.218 \\
\hline 215.99 & 4.459 & 3.622 & 3.584 & 3.602 \\
\hline 297.42 & 4.970 & 4.440 & 4.395 & 4.407 \\
\hline 596.65 & 5.531 & 6.921 & 6.923 & 6.939 \\
\hline 1000.00 & 5.671 & 9.733 & 9.740 & - \\
\hline 10000.00 & 5.751 & * & 44.341 & - \\
\hline 100000.00 & 5.752 & $*$ & 181.343 & - \\
\hline Average error & $12.55 \%$ & $0.23 \%$ & $0.31 \%$ & - \\
\hline \multicolumn{5}{|c|}{ Spacing between axes: $79.45 \mathrm{~mm}$} \\
\hline 0 & 1.000 & 1.000 & 1.000 & 1.000 \\
\hline 25.20 & 1.065 & 1.062 & 1.061 & 1.063 \\
\hline 50.35 & 1.233 & 1.206 & 1.205 & 1.208 \\
\hline 75.42 & 1.452 & 1.378 & 1.377 & 1.378 \\
\hline 100.43 & 1.673 & 1.552 & 1.550 & 1.554 \\
\hline 215.82 & 2.337 & 2.189 & 2.187 & 2.196 \\
\hline 296.92 & 2.534 & 2.523 & 2.520 & 2.519 \\
\hline 401.85 & 2.659 & 2.891 & 2.889 & 2.890 \\
\hline 500.97 & 2.719 & 3.198 & 3.196 & 3.199 \\
\hline 597.50 & 2.753 & 3.469 & 3.468 & 3.468 \\
\hline 1000.00 & 2.807 & 4.416 & 4.412 & - \\
\hline 10000.00 & 2.838 & $*$ & 13.498 & - \\
\hline 100000.00 & 2.838 & $*$ & 42.588 & - \\
\hline Average error & $6.59 \%$ & $0.10 \%$ & $0.13 \%$ & - \\
\hline
\end{tabular}

Bold numbers indicate that $x_{s}>2.8$, so (25) is inaccurate

* indicates that $x>12$, so Arnold's formula cannot be applied 
The $R_{a d} / R_{d c}$ values for the last three frequencies shown in Table 5 are not given in [18].

Results shown in Table 5 prove that results from both FEM simulations and Arnold's formula (25) produce very close results, which are very similar to the experimental ones. However, the inaccuracy of the IEC 60287-1-1 formula grows with increasing frequency values. For example, when the conductors are spaced $32.56 \mathrm{~mm}$, at $25 \mathrm{~Hz}$ the relative error with respect the Arnold's formula is about $0.3 \%$ whereas at 50 $\mathrm{Hz}$ and $600 \mathrm{~Hz}$ it is about $6.0 \%$ and $20.1 \%$, respectively. However, Arnold's formula is difficult to apply when compared with the one suggested by the IEC 60287-1-1 standard.

Results presented in Table 5 assume that the two conductors carry currents of opposite polarity. However, when dealing with conductors carrying currents of the same polarity the results can change significantly, as shown in Table 6.

\section{Table 6}

$R_{a c} / R_{d c}$ values for two parallel solid round $\mathrm{Cu}$ conductors with radius $16 \mathrm{~mm}$ calculated at $1000 \mathrm{~Hz}$ as a function of the spacing between axes carrying AC currents of the same and different polarities

\begin{tabular}{ccc}
\hline $\begin{array}{c}\text { Spacing } \\
(\mathbf{m m})\end{array}$ & $\begin{array}{c}\text { FEM } \\
++\end{array}$ & $\begin{array}{c}\text { FEM } \\
+-\end{array}$ \\
\hline 32.1 & 5.356 & 10.337 \\
40.0 & 5.037 & 6.110 \\
50.0 & 4.748 & 5.079 \\
60.0 & 4.567 & 4.707 \\
70.0 & 4.451 & 4.513 \\
80.0 & 4.369 & 4.403 \\
90.0 & 4.313 & 4.330 \\
100.0 & 4.274 & 4.280 \\
200.0 & 4.135 & 4.136 \\
500.0 & 4.097 & 4.097 \\
\hline
\end{tabular}

Results summarized in Table 6 show that the resistance ratio increases when decreasing the spacing between conductors' axes, due to the combination of both skin and proximity effects, thus increasing power losses in conductors. Results in Table 6 also prove that the resistance ratio is higher in conductors carrying current of different polarity than in conductors carrying currents of the same polarity. This is because when the two conductors carry currents of opposite polarity, the current density is concentrated in the conductors' sides closer to the other conductor, and this effect is enhanced when the conductors are very near each 
other. When dealing with conductors carrying currents of the same polarity, the current density is concentrated in the regions farthest away from both conductors, so the current density and thus the resistance ratio are less affected by the distance between conductors. Table 6 also shows that when the two parallel conductors are spaced enough, the resistance ratio tends to that of a single isolated conductor which is 4.09 at $1000 \mathrm{~Hz}$ for a copper conductor with a radius of $16 \mathrm{~mm}$.

\subsection{AC resistance ratio of three parallel round solid conductors carrying three-phase currents}

In the case of three neighboring rectilinear parallel conductors carrying three phase currents, both proximity and skin effects can severely increase the AC resistance of the conductors. Although there isn't an exact formula for this geometry, the IEC 60287-1-1 standard [10] provides an approximate formula to calculate the $R_{a c} / R_{d c}$ resistance ratio for this configuration. This section compares the results for three parallel conductors carrying three-phase AC currents provided by the approximated formula (25) with those outputted by the FEM model.

Table 7 summarizes the results attained for three parallel solid round copper conductors with radius 10 $\mathrm{mm}$ and spaced $25 \mathrm{~mm}$ between axes, arranged in a triangular formation and carrying three-phase balanced currents.

\section{Table 7}

$R_{a c} / R_{d c}$ values for three parallel solid round $\mathrm{Cu}$ conductors arranged in a triangular formation with radius 10 $\mathrm{mm}$, spaced $25 \mathrm{~mm}$ between axes carrying three-phase balanced currents

\begin{tabular}{ccc}
\hline $\begin{array}{c}\text { Frequency } \\
\text { (Hz) }\end{array}$ & $\begin{array}{c}\text { IEC 60287-1-1 } \\
(\mathbf{2 5}),(\mathbf{2 8})\end{array}$ & FEM \\
\hline 0.0 & 1.000 & 1.000 \\
25.0 & 1.026 & 1.026 \\
50.0 & 1.098 & 1.097 \\
75.0 & 1.201 & 1.197 \\
100.0 & 1.318 & 1.310 \\
200.0 & $\mathbf{1 . 7 7 7}$ & 1.769 \\
500.0 & $\mathbf{2 . 5 4 1}$ & 2.829 \\
1000.0 & $\mathbf{2 . 8 7 4}$ & 4.021 \\
10000.0 & $\mathbf{3 . 0 2 9}$ & 12.997 \\
100000.0 & $\mathbf{3 . 0 3 1}$ & 41.732 \\
\hline
\end{tabular}

Bold numbers indicate that $x_{\mathrm{s}}>2.8$, so (25) is inaccurate 
Results summarized in Table 7 indicate that for the considered triangular formation, the results provided by the IEC formula and FEM simulations are very close for frequencies up to $200 \mathrm{~Hz}$, however for frequencies above $200 \mathrm{~Hz}$ the IEC approximate formula becomes inaccurate.

\subsection{AC resistance of two parallel round tubular conductors}

In this section the AC resistance of two neighboring parallel tubular conductors is evaluated. To this end, experimental results of two parallel tubular conductors with $r_{l}=4.74 \mathrm{~mm}$ and $r_{2}=6.33 \mathrm{~mm}$ published in [25] are compared with those provided by the approximated formula (25) suggested by the IEC 60287-1-1 standard and FEM simulations. These results are shown in Table 8.

\section{Table 8}

$R_{a c} / R_{d c}$ values for two round tubular $\mathrm{Cu}$ conductor with $r_{1}=4.74 \mathrm{~mm}$ and $r_{2}=6.33 \mathrm{~mm}$ spaced $13 \mathrm{~mm}$ and $0.1 \mathrm{~mm}$ carrying AC currents of opposite polarity

\begin{tabular}{cccc}
\hline $\begin{array}{c}\text { Frequency } \\
(\mathbf{H z})\end{array}$ & $\begin{array}{c}\text { IEC 60287-1-1 } \\
(\mathbf{2 5 )}\end{array}$ & FEMM & $\begin{array}{c}\text { Measured } \\
{[\mathbf{2 5}]}\end{array}$ \\
0 & Conductors spaced 13 mm & \\
320 & 1.000 & 1.000 & 1.000 \\
658 & 1.003 & 1.011 & 1.013 \\
994 & 1.012 & 1.039 & 1.039 \\
1482 & 1.028 & 1.068 & 1.067 \\
1987 & 1.059 & 1.103 & 1.102 \\
2482 & 1.100 & 1.133 & 1.132 \\
3004 & 1.147 & 1.161 & 1.160 \\
3467 & 1.199 & 1.191 & 1.188 \\
3915 & 1.247 & 1.218 & 1.216 \\
4540 & 1.295 & 1.247 & 1.241 \\
5180 & 1.360 & 1.289 & 1.288 \\
Average error & 1.425 & 1.336 & 1.327 \\
\hline \multicolumn{4}{c}{ Conductors spaced 0.1 mm } \\
488 & 1.000 & 1.000 & - \\
1384 & 1.021 & 1.104 & 1.000 \\
2040 & 1.160 & 1.495 & 1.524 \\
3030 & 1.316 & 1.743 & 1.787 \\
3930 & 1.585 & 2.052 & 2.104 \\
5040 & 1.824 & 2.299 & 2.364 \\
Average error & 2.085 & 2.585 & 2.630 \\
\hline
\end{tabular}


Results summarized in Table 8 prove that results from both FEM simulations are quite similar to the experimental ones. However, the inaccuracy of the IEC 60287-1-1 formula grows when increasing the frequency, especially when both conductors are very close each other, since the proximity effect is more pronounced.

Results presented in Table 8 assume that the two conductors carry currents of opposite polarity. However, when dealing with conductors carrying currents of the same polarity the results can change significantly, as proved in Table 9, which show the important effect of the polarity, especially when both conductors are very close each other.

\section{Table 9}

$R_{a c} / R_{d c}$ values for two round tubular $\mathrm{Cu}$ conductor with $r_{l}=4.74 \mathrm{~mm}$ and $r_{2}=6.33 \mathrm{~mm}$ spaced $13 \mathrm{~mm}$ and $0.1 \mathrm{~mm}$ carrying AC currents of the same polarity

\begin{tabular}{|c|c|c|c|c|c|}
\hline $\begin{array}{c}\text { Frequency } \\
(\mathrm{Hz})\end{array}$ & $\begin{array}{c}\text { FEM } \\
++ \\
\end{array}$ & $\begin{array}{c}\text { FEM } \\
+-\end{array}$ & $\begin{array}{c}\text { Frequency } \\
(\mathrm{Hz})\end{array}$ & $\begin{array}{c}\text { FEM } \\
++\end{array}$ & $\begin{array}{c}\text { FEM } \\
+-\end{array}$ \\
\hline \multicolumn{3}{|c|}{ Conductors spaced $13 \mathrm{~mm}$} & \multicolumn{3}{|c|}{ Conductors spaced $0.1 \mathrm{~mm}$} \\
\hline 0 & 1.000 & 1.000 & 0 & 1.000 & 1.000 \\
\hline 320 & 1.011 & 1.011 & 488 & 1.089 & 1.104 \\
\hline 658 & 1.038 & 1.039 & 1384 & 1.264 & 1.495 \\
\hline 994 & 1.064 & 1.068 & 2040 & 1.326 & 1.743 \\
\hline 1482 & 1.094 & 1.103 & 3030 & 1.398 & 2.052 \\
\hline 1987 & 1.120 & 1.133 & 3930 & 1.464 & 2.299 \\
\hline 2482 & 1.145 & 1.161 & 5040 & 1.554 & 2.585 \\
\hline 3004 & 1.172 & 1.191 & & & \\
\hline 3467 & 1.198 & 1.218 & & & \\
\hline 3915 & 1.225 & 1.247 & & & \\
\hline 4540 & 1.265 & 1.289 & & & \\
\hline 5180 & 1.311 & 1.336 & & & \\
\hline Average error & $1.22 \%$ & - & Average error & $21.26 \%$ & - \\
\hline
\end{tabular}

\section{CONCLUSION}

This paper has analyzed the behavior of different exact and approximate formulas to calculate the AC resistance of electrical conductors carrying AC currents in a wide frequency range. The results provided by these formulas have been compared with those found in published experimental data and those obtained through FEM simulations. Results presented show the limitations of the analyzed formulas, since they only can deal with a limited number of configurations and the superior performance of the FEM simulations due 
to their accuracy and wide range of electrical frequencies and geometries that the FEM method allows analyzing. The analyses carried out also show that the approximated formula suggested by the IEC 602871-1 standard can lead to inaccurate results even at power frequency for some conductors' configurations.

\section{REFERENCES}

[1] W. I. Middleton and E. W. Davis, Skin effect in large stranded conductors at low frequencies, J. Am. Inst. Elect. Eng., 40-9 (1921) 757-763

[2] V. T. Morgan, The current distribution, resistance and internal inductance of linear power system conductors - A review of explicit equations, IEEE Trans. Power Deliv., 28-3 (2013) 1252-1262.

[3] J. -R. Riba, Calculation of the ac to dc resistance ratio of conductive nonmagnetic straight conductors by applying FEM simulations, Eur. J. Phys. (2015), accepted for publication

[4] M. M. Al-Asadi, A. P. Duffy, A. J. Willis, K. Hodge, and T. M. Benson, A simple formula for calculating the frequency-dependent resistance of a round wire, Microw. Opt. Techn. Let., 19- 2 (1988) 84-87.

[5] A. Reatti and F. Grasso, Solid and Litz-Wire Winding Non-linear Resistance Comparison, Proc. 43rd IEEE Midwest Symp. on Circuits and Systems, Lansing MI, Aug 8-11, 2000, pp. 466-469.

[6] Dai, D.; Zhang, X., Wang, J., Calculation of AC resistance for stranded single-core power cable conductors, IEEE Trans. Magn., 50-11 (2014), 6301104.

[7] A. A. P. Silva, J. M. B. Bezerra, Applicability and limitations of ampacity models for HTLS conductors, Electr. Pow. Syst. Res. 93 (2012) 61- 66.

[8] A. Reatti and M. K. Kazimierczuk, Comparison of Various Methods for Calculating the AC Resistance of Inductors, IEEE Trans. Magn. 38-3 (2002) 1512-1518.

[9] S. J. Haefner, Alternating-current resistance of rectangular Conductors, Proc. Inst. Radio Engin., 25-4 (1937) 434-447.

[10]IEC 60287-1-1, Electric cables - Calculation of current rating - Part 1: Current rating equations (100\% load factor) and calculation of losses - Section 1: General, 2006.

[11]W. T. Weeks, L. L. Wu, M. F. McAllister, A. Singh, Resistive and inductive skin effect in rectangular conductors, IBM J. Res. Dev., 23- 6 (1979) 652 - 660.

[12]X. Nan, C. R. Sullivan, An improved calculation of proximity-effect loss in high-frequency windings of round conductors, IEEE Power Electr. Specialists Conference, June 2003, pp. 853-860.

[13]I. Lope, C. Carretero, J. Acero. Alonso, and J. M. Burdío, AC power losses model for planar windings with rectangular cross-sectional conductors, IEEE Trans. Power Electron. 29-1 (2014) 23-28.

[14]M. T. Villéna, J. Letosa, A. Nogués, R. Murillo, Procedure to accelerate calculations of additional losses in transformer foil windings, Electr. Pow. Syst. Res. 95 (2013) 85-89.

[15]G. S. Smith, A simple derivation for the skin effect in a round wire, Eur. J. Phys. 35 (2014) 1-13.

[16]U.S. Gudmundsdottir, Proximity effect in fast transient simulations of an underground transmission cable, Electr. Pow. Syst. Res. 115 (2014) 50-56.

[17]F. W. J. Olver, D. W. Lozier, R. F. Boisvert, and C. W. Clark, Handbook of Mathematical Functions, Cambridge University Press, Cambridge, 2010.

[18]A. H. M. Arnold, The alternating-current resistance of parallel conductors of circular cross-section, J. Inst. Electr. Eng. 77-463 (1935) 49-58. 
[19]J. Hernández-Guiteras, J. -R. Riba, L. Romeral, Improved design of an extra-high-voltage expansion substation connector through magnetic field analysis, Simul. Model. Pract. Theory 43 (2014) 96-10.

[20]J. Hernández-Guiteras, J. -R. Riba, L. Romeral, Redesign process of a 765 kVRMS AC substation connector by means of 3D-FEM simulations, Simul. Model. Pract. Theory 42 (2014) 1-11.

[21]D. C. Meeker. (2014, Nov. 24). Finite Element Method Magnetics [Online]. Available: http://www.femm.info

[22]J. Larsson, Electromagnetics from a quasistatic perspective, Am. J. Phys. 75-3 (2007) 230-239.

[23]British Electrical and Allied Industries Research Association, Copper losses in large cables at power frequencies, J. Inst. Electr. Eng. 76-459 (1935) 299-322.

[24]A. E. Kennelly, Skin-effect resistance measurements of conductors at radio-frequencies up to 100,000 cycles per second, Proc. of the Institute of Radio Engineers, 4-6 (1917) 523-574;

[25]A.E. Kennelly, F. Laws, P. Pierce, Experimental researches on skin effect in conductors, Trans. Am. Inst. Electr. Eng. 34 (1915) 1953-20013.

[26]D. Lovric, V. Boras, S. Vujevic, Accuracy of approximate formulas for internal impedance of tubular cylindrical conductors for large parameters, Prog. Electromagn. Res. M, 16 (2011) 171-184. 